\title{
EPIDEMIOLOGICAL AS WELL AS MICROBIOLOGICAL PROFILE OF SUPPURATIVE KERATITIS AND IT'S OUTCOME: A PROSPECTIVE HOSPITAL BASED STUDY FROM EASTERN NEPAL
}

\author{
Patel S. ${ }^{1}$, Badhu B.P. ${ }^{2}$, Khanal B. ${ }^{3}$, Chaudhary S. ${ }^{4}$, Arya S.K. ${ }^{5}$, Dhakhwa K. ${ }^{1}$
}

\begin{abstract}
INTRODUCTION: Suppurative keratitis is the second most common cause of monocular blindness after unoperated cataract in some tropical developing countries. The etiological and epidemiological pattern of suppurative keratitis varies significantly with geographical region, patient's population, health of the cornea. The present study was carried out to explore the epidemiological pattern, microbiological profile and treatment outcome of suppurative keratitis at a tertiary care center in Eastern Nepal.
\end{abstract}

MATERIAL AND METHODS: It was a prospective cohort study carried out in patients presented with suppurative keratitis from 1 November 2007 to 31 October 2008. Detailed clinical history, general physical examination and meticulous ophthalmological examination were carried out in all the subjects as per pro-forma. Corneal scraping was performed under aseptic condition. Material obtained from corneal scrapping was smeared on three separate glass slides for Gram stain, Giemsa stain and $\mathrm{KOH}$ wet mount. Material obtained from scraping was inoculated directly on to sheep's blood agar, chocolate agar, BHI, SDA media and sent for microbiological examination. Patients were followed up after 1 week, 2 weeks and 1 month of starting therapy.

RESULTS: Sixty patients with suppurative keratitis were evaluated. 39 (65\%) patients were male and 21 (35\%) patients were female. Mean age of the patients was $41.53 \pm 17.86$ year. Mean duration of illness was $20.23 \pm 18.62$ days. History of ocular trauma was present in $35(58.33 \%)$ patients. corneal ulcer was more often seen during summer (May-July) and winter (November-January) months. During culture, growth was seen in $33(55 \%)$ patients. Fifteen cases $(45.45 \%)$ had a pure bacterial growth, $10(30.30 \%)$ patients had pure fungal growth and $8(24.25 \%)$ cases had mixed bacterial and fungal growth. Staphylococcus aureus was isolated in 15 (25\%) cases. Among fungi Aspergillus flavus, Aspergillus fumigatus, Fusarium were the frequent isolates, found in $4(6.67 \%), 3(5 \%), 3(5 \%)$ cases. Staphylococcus aureus was found in all the cases with mixed growth. Out of 60 patients $49(81.67 \%)$ patients achieved, good out come in form of complete healing of corneal ulcer. Poor outcome was seen in $11(18.33 \%)$ patients.

CONCLUSION: Suppurative keratitis was more commonly seen in middle aged male from rural areas, involved in agriculture related work. Staphylococcus aureus was the most common bacterial isolates. Aspergillus was the most common fungal species. With treatment majority of patients (81.67\%) had good out come in form of complete healing of the corneal ulcer.

KEY WORDS: Suppurative Keratitis, Corneal Ulcer, Ocular Trauma, Fungal Keratitis, Corneal Scar, PKP

1. Ophthalmologist, Lumbini Eye Institute, Bhairahawa, Nepal

2. Professor, Department of Ophthalmology, BPKIHS, Dharan, Nepal

3. Professor, Department of Microbiology, BPKIHS, Dharan, Nepal

4. Assistant Professor, Department of Internal Medicine, Universal College of Medical Sciences \& Teaching Hospital, Bhairahawa, Nepal

5. Professor, Department of Ophthalmology, GMC, Chandigarh, India

For Correspondence

Dr. Sushila Patel, M.D.,

Ophthalmologist,

Lumbini Eye Institute,

Bhairahawa, Nepal

E-mail:drsushilapatel@yahoo.com 


\section{INTRODUCTION}

The World Health Organization (WHO) has recognized that corneal blindness resulting from suppurative keratitis is emerging as a leading cause of visual disability. ${ }^{1}$ Suppurative keratitis is an important preventable cause of monocular blindness worldwide. ${ }^{2-3}$ Corneal infections are the second most common cause of monocular blindness after unoperated cataract in some tropical developing countries. ${ }^{4}$ In the developing world, corneal ulcers appear to be occurring in epidemic proportions, being ten times more common than in developed countries. ${ }^{2}$ The incidence of corneal ulcer in South East Asia is especially high compared with other parts of the world. The incidence of microbial keratitis varies from 11 per 100,000 persons / year in the United States to 799 per 100,000 persons / year in Nepal.

The etiological and epidemiological pattern of corneal ulceration varies significantly with patient's population, health of the cornea, geographical region. ${ }^{6}$ Trauma is a far more common predisposing factor for suppurative keratitis in developing countries. Non-surgical trauma to the eye accounted for $48.6-65.4 \%$ of all corneal ulcers in the developing countries like Nepal and India, ${ }^{7}$ whereas it accounted for only $27 \%$ of all cases at a large country trauma referral centre in the United states. ${ }^{8}$ Hence, understanding of the current status of the regional epidemiological features, risk factors, the presence of ocular and/or systemic co-morbidities, occupational status is important in the prevention and management of suppurative keratitis.

The common modes of ocular trauma in developing countries are vegetative matters like paddy, paddy stalk, jute plants during agricultural work. Other significant agents are twig of a tree, flying insect, dirt, mud, sand etc. Certain ocular conditions like chronic dacryocystitis, dry eye, trichiasis, entropion, bullous keratopathy, spheroidal degeneration, lagophthalmos and certain systemic diseases like diabetes mellitus, leprosy are also known to be associated with suppurative keratitis. ${ }^{9}$ In the recent years there has been an increase in the prevalence of infective keratitis in the developing world due to the inadvertent use of steroids and antibiotics, the later producing resistant strains, as well as improper and delayed diagnosis.

For proper management of suppurative keratitis it is important to undertake comprehensive surveys investigating the predisposing and demographic factors contributing the corneal ulcerations. The present study was carried out to explore the demographic pattern, microbiological profile and treatment outcome of suppurative keratitis at a tertiary care center in Eastern Nepal.

\section{MATERIAL AND METHODS}

It was a prospective cohort study. All the patients with suppurative corneal ulcer presenting inpatient and outpatient clinics of the department of ophthalmology, B. P. Koirala Institute of Health Sciences, Dharan from 1 November 2007 to 31 October 2008 were included in the study. Patients with typical or suspected viral ulcers, Acanthamoeba keratitis were excluded from the study. Likewise noninfectious peripheral ulcerative keratitis, atheromatous ulcer, vernal keratopathy, Interstitial keratitis and any other noninfectious corneal ulcer were also excluded from the study

After obtaining a written informed consent, detailed clinical history, general physical examination and meticulous ophthalmological examination was carried out in all the subjects as per predesigned pro-forma. History regarding onset of various symptoms like pain, redness, photophobia, watering, discharge and diminution of vision was taken. History of predisposing factors like corneal trauma, contact lens wear and prolonged use of topical or systemic steroids, corneal surgery was taken. History of systemic illness like Diabetes, chronic debilitating illness especially malnutrition, collagen vascular disease, immunocompromised status, tuberculosis, leprosy was taken. Visual acuity of both the eyes was measured with Snellen chart. Detailed corneal examination was done for morphology of corneal ulcer. The size of corneal ulcer was measured after staining with fluorescein using the graduated slit beam of a Haag-Streit 900 slit lamp. Firstly the longest dimension of the defect was determined and then dimension perpendicular to the first was measured in millimeters. The location of the ulcer was determined according to the zone i.e. central, nasal, inferior, temporal and superior and corneal diagram was drawn. Stromal infiltrate was looked for their colour, depth and margin along with surrounding corneal haze. The corneal ulcer depth was evaluated as $<20 \%, 20-50 \%$, or $>50 \%$ of the total corneal thickness. Corneal vascularization was looked for and labeled as deep or superficial.

Anterior chamber was examined for presence of cells, flare, and hypopyon as per Hogan's classification. Anterior chamber depth was seen on slit lamp to look for any abnormality. Corneal ulcer was graded according to the table given below. (Table1) 
Table1: Grading scheme for corneal ulcers. ${ }^{10}$

\begin{tabular}{|l|c|c|c|}
\hline & Mild & Moderate & Severe \\
\hline $\begin{array}{l}\text { Size of epithelial } \\
\text { defect (mm) }\end{array}$ & Less than 2 & 2 to 5 & Over 5 \\
\hline Depth of ulcer (\%) & Less than 20 & 20 to 50 & Over 50 \\
\hline Depth of Infiltrate & Superficial & $\begin{array}{c}\text { Dense, } \\
\text { mid-stromal }\end{array}$ & $\begin{array}{c}\text { Dense, } \\
\text { Past mid-stroma }\end{array}$ \\
\hline Sclera & Not involved & Not involved & May be involved \\
\hline
\end{tabular}

Corneal scraping was performed under aseptic condition from leading edge of corneal ulcer by using a flamed sterilized Kimura spatula. Scrapping was performed after topical anesthesia with 4\% Xylocaine with out preservatives. Material obtained from corneal scrapping was smeared on three separate glass slides: one for Gram stain, another for Giemsa stain and the other for $\mathrm{KOH}$ wet mount. Direct microscopic examination was performed in Gram, Giemsa stained smear and $\mathrm{KOH}$ wet mount for the presence of inflammatory cells, bacterial and fungal elements. Material obtained from scraping was inoculated directly on to sheep's blood agar, chocolate agar, brain heart infusion broth (BHI), Sabouraud dextrose agar (SDA) media. For bacterial cultures, plates and media were submitted to microbiology department for further processing. The plates were incubated aerobically at $37^{\circ} \mathrm{C}$ in microbiology laboratory. Cultures on blood agar and chocolate agar were evaluated at 24 hours and 48 hours, and then discarded if there was no growth. From BHI broth with inoculated material, the incubation and identification of growth was performed and examined in the similar fashion. Subcultures were done onto blood agar and MacConkey agar. The bacterial colony if any was further processed and identified following standard microbiological technique. Bacterial cultures were considered positive only if growth of the organism has been demonstrated in both media or there is semi confluent growth at the site of inoculation on one media with identification of morphological characteristics of similar organism on Gram stain. For fungal cultures, the materials were inoculated onto SDA media and incubated at room temperature, examined daily and discarded after 2 weeks if there was no growth. Fungi were identified by their colony characteristics on SDA and by the morphological appearance of the spores in some cases by slide culture method. If by microscopy in $\mathrm{KOH}$ mount preparation, hyphae were observed in corneal smear, but failed to grow in culture, the causative organism was reported as fungal. For the entire bacterial isolates antimicrobial susceptibility test was performed by Kirby Bauer Disc diffusion method. Processing, reading of the results and zone size interpretation has been performed as per NCCLS(CLSI) guidelines. ${ }^{11}$ All the bacterial isolates were tested for antimicrobial sensitivity with Amikacin, Gentamicin, Tobramycin, Cefazolin, Ciprofloxacin, Ofloxacin and Erythromycin. Initial therapy was decided on the basis of smear report. If it was negative, then on the basis of patient's history and clinical examination, monotherapy in the form of antibacterial or antifungal had been started.

For mild bacterial corneal ulcer- Fluoroqinolones (Ofloxacin) eye drops had been started every hour for 2-3 days. Then it was tapered according to clinical response of the corneal ulcer. For moderate to severe bacterial corneal ulcer- Duotherapy consisting of fortified cefazolin 5\% and tobramycin $1.36 \%$ eye drops were used. For mild fungal corneal ulcer- 5\% Natamycin eye drops were started every hour and then tapered according to clinical response. For moderate to severe fungal corneal ulcer- 5\% Natamycin eye drops and systemic Itraconazole (100 mg BD) or Fluconazole (150 BD) were given for two weeks. All the patients with bacterial or fungal corneal ulcer were given Atropine 1\% eye drop, antiglaucoma, tab vitamin C along with antibacterial or antifungal therapy. In severe corneal ulcer not responding to the above treatment or impending corneal perforation, treatment was modified in the form of systemic antibacterial or antifungal therapy. For perforated corneal ulcer, bandaged contact lens or therapeutic penetrating keratoplasty was done. Intracameral Amphotericin B was given in non responding deep severe fungal corneal ulcers. Patients were followed up after 1 week, 2 week and 1 month of starting therapy. Clinical response had been evaluated in the form of visual acuity, healing of corneal ulcer and complications if any at each follow up visits. Treatment outcome had been evaluated in form of signs of healing, final visual acuity achieved, surgical intervention required or not and complication if any.

The collected data were entered into Microsoft Excel Spreadsheet and analyzed using SPSS ver 11.5 (PC) / EpiInfo (CDC, Atlanta, GA, USA). Means and standard deviation of age of the patients and duration of illness was calculated. Chisquare $\left(\chi^{2}\right)$ test was used for comparing treatment outcome and corneal perforation with gender of patient, previous treatment, Residence of patient, presence or absence of trauma, size of corneal ulcer, depth of corneal ulcer, depth of stromal infiltrates, presence or absence of hypopyon, gram stain, Giemsa stain, $\mathrm{KOH}$ mount, presence or absence of growth in culture. 


\section{RESULTS}

A total of 60 patients with suppurative corneal ulcer were analyzed in this study. The average age of the patients was $41.53 \pm 17.86$ years ranging from 5-83 years. Most of the patients were in between $30-60$ years of age $(61.67 \%$ of all patients). Male were affected more than female. In our study $39(65 \%)$ patients were male and $21(35 \%)$ patients were female. Most of patients were from surrounding districts and neighboring Indian state of Bihar. Majority of the patients were from Sunsari district. Twenty six (43.33\%) patients were from Nepal and 34 (56.67\%) patients were from Indian state of Bihar. Majority of the patients were from rural areas. Forty four $(73.3 \%)$ were from rural areas and $16(26.7 \%)$ were from urban areas. Agricultural workers 22 (33.67\%), housewives 17 $(28.33 \%)$ and students $9(15 \%)$ were the three most common groups comprising $48(80 \%)$ of the total study population. Others were ex-serviceman 4 (6.67\%), labourer $4(6.67 \%)$, serviceman $3(5 \%)$, businessman 1(1.67\%). Patients came with corneal ulcer in every month of the year but more patients came during summer (May-July) and winter (NovemberJanuary) months. (Figure 1)

\section{Figure 1: Seasonal vatiation of corneal ulcer}

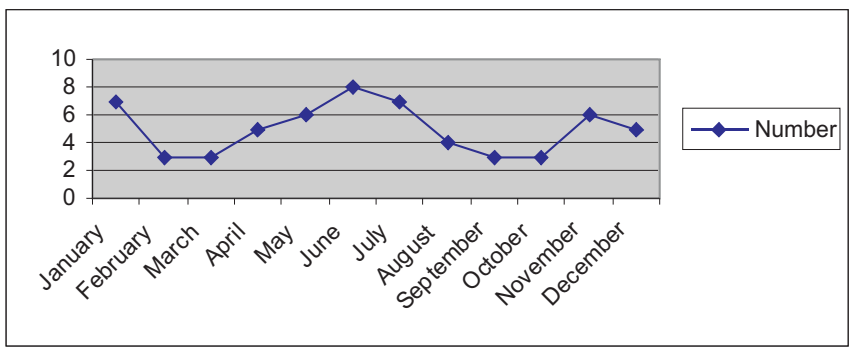

Mean duration of illness was $20.23 \pm 18.62$ days ranging from 2 to 90 days. Right eye and left eye were involved equally. There was no difference in the two eyes in terms of laterality. There was history of ocular trauma in $35(58.33 \%)$ patients and in 25 $(41.67 \%)$ patients no predisposing factor was found. Vegetative trauma during agricultural work was the commonest mode of trauma andwas present in $15(25 \%)$ patients. Various other agents responsible for trauma were wooden stick $5(8.33 \%)$, finger nail $5(8.33 \%)$, Insect 4 (6.67\%), Mud 3 (5\%), Iron Nail/Metal Wire 2 (3.34\%) and trichiasis $1(1.67 \%)$. Two patients were found to be diabetic, one patient was hypertensive. One patient was getting treatment for pulmonary tuberculosis and one patient was pregnant. Majority of patients $49(81.67 \%)$ had already received treatment from outside. At the time of presentation visual acuity was taken with the help of Snellen chart. Vision in the involved eye varied from 6/6 to NPL. Most of the patients presented with very low vision. Forty four patients had vision between finger counting to no perception of light. (Table 2)

Table 2: Vision of involved eye during first visit

\begin{tabular}{|c|c|}
\hline Vision of involved eye & Numbers (\%) \\
\hline $6 / 6-6 / 12$ & $6(10 \%)$ \\
\hline $6 / 18-6 / 36$ & $2(3.33 \%)$ \\
\hline $6 / 60-1 / 60$ & $8(13.33 \%)$ \\
\hline CF PL & $43(71.67 \%$ \\
\hline NPL & $1(1.67 \%)$ \\
\hline
\end{tabular}

Most common position of ulcers was central which was found in $37(61.7 \%)$ of the patients; followed by inferior, seen in 12 $(20 \%)$ of the patients. Temporal position was found in 7 $(11.7 \%)$ patients, superior and nasal position was seen in 3 $(5 \%)$ and $1(1.6 \%)$ patients. Size of corneal ulcers was varying from $0.4 \mathrm{~mm}$ to $11 \mathrm{~mm}$. Ulcers were graded according to size in to mild, moderate and severe. Ten patients (16.67\%) were having ulcer size $<2 \mathrm{~mm}, 20$ patients $(33.33 \%)$ had size between $2-5 \mathrm{~mm}$ and 30 patients $(50 \%)$ had size $>5 \mathrm{~mm}$ in diameter. Depth of ulcer was $<20 \%$ in $12(20 \%)$ cases, $20-50 \%$ in $23(38.33 \%)$ cases, and $>50 \%$ in $25(41.67 \%)$ cases. Superficial infiltrates were seen in $14(23.33 \%)$ patients, Infiltration up to mid stroma was seen in $25(41.67 \%)$ patients and deep infiltrates were seen in $21(35 \%)$ patients. Corneal ulcers were graded in mild, moderate and severe depending upon size of epithelial defect (mm), depth of ulcer (\%), depth of infiltrate and scleral involvement. Ulcer was of mild grade in $13(21.7 \%)$ patients, moderate grade in $22(36.6 \%)$ patients and severe in $25(36.6 \%)$ patients. Hypopyon was present in 29 $(48.3 \%)$ patients and it was absent in $31(51.7 \%)$ patients.

\section{MICROBIOLOGICAL EVALUATION}

Out of 60 patients of suppurative corneal ulcer analyzed, in culture growth was seen in $33(55 \%)$ patients. Among them fifteen cases $(45.45 \%)$ had a pure bacterial growth, 10 $(30.30 \%)$ patient had pure fungal growth and $8(24.25 \%)$ cases had mixed bacterial and fungal growth. (Figure 2)

\section{Figure 2: Culture results}

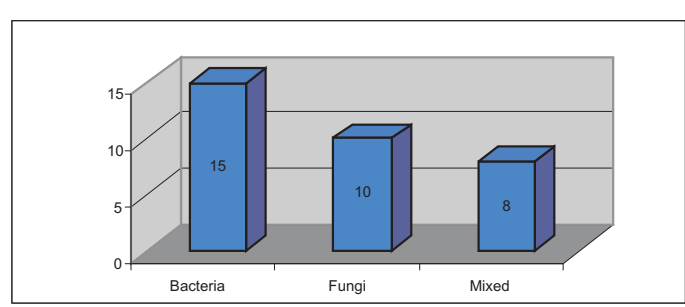


In Gram staining bacteria was seen in $4(6.67 \%)$ cases. Gram positive cocci were seen in $3(5 \%)$ cases and Gram negative bacilli were seen in $1(1.67 \%)$ case. In Giemsa staining fungal hyphae were seen in $3(5 \%)$ cases. In $10 \% \mathrm{KOH}$ mount, fungal elements were seen in $5(8.33 \%)$ cases. Among them 4 $(6.67 \%)$ were septate fungi and $1(1.67 \%)$ thick branched septate fungi. Bacteria were isolated in a total of 23 cases, of which $15(25 \%)$ appeared as pure bacterial growth and 8 $(13.33 \%)$ had mixed growth with fungus. Staphylococcus aureus was isolated in $15(25 \%)$ cases, among them in 4 $(6.67 \%)$ cases it was single isolate and in $11(18.33 \%)$ it was a mixed growth along with fungus. Other Gram positive bacteria isolated in culture were Streptococcus pneumoniae 2 (3.33\%), Coagulase neg Staphylococcus 1 (1.67\%), Bacillus 1 (1.67\%), Diphtheroids 1 (1.67\%). Gram negative bacterial isolates were Acinetobacter 3 (5\%), Pseudomonas 2 (3.33\%), E coli 2 (3.33\%), Klebsiella 1 (1.67\%), Citrobacter 1 (1.67\%), Enterobacter 1 (1.67\%). Among fungi Aspergillus flavus, Aspergillus fumigatus and Fusarium were the frequent isolates, found in $4(6.67 \%), 3(5 \%)$ and $3(5 \%)$ cases respectively. Other fungi which were isolated were Aspergillus niger 2 (3.33\%), Auerobasidium 2 (3.33\%), Candida 1 (1.67\%), Rhinocladiella aqua 1 (1.67\%), Succinia 1 (1.67\%), Trichophyton concentrica 1 (1.67\%). Eight $(13.33 \%)$ cases had mixed bacterial and fungal growth. Staphylococcus aureus was found in all the cases with mixed growth. In four cases there were mixed bacterial growth.

\section{TREATMENT OUTCOME}

Treatment was considered successful where the corneal infiltrates resolved (with or without consequential scarring) and where the overlying corneal epithelium healed completely. Clinical outcome was deemed to be poor if corneal perforation developed despite antimicrobial therapy, Contact bandage lens or temporary tarsorrhaphy was needed to treat a persistent epithelial defect, where a penetrating keratoplasty (PKP) was performed for visual restoration or for infection control, or where an eye destructive procedure (enucleation or evisceration) was necessary. Out of 60 patients, 49 (81.67\%) patients achieved good out come in form of complete healing of corneal ulcer. Among them in 7 (11.67\%) patients there was minimal corneal scarring whereas in $42(70 \%)$ patients healing of corneal ulcer occurred with dense leucomatous opacity. (Figure3)

Figure 3: Treatment outcome

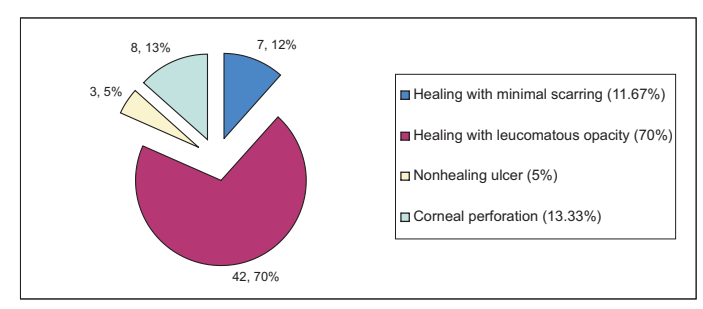

Poor outcome was seen in $11(18.33 \%)$ patients. Among them ulcer was nonhealing in $3(5 \%)$ patients and corneal perforation occurred in $8(13.33 \%)$ patients. Among 3 cases of nonhealing corneal ulcers, therapeutic penetrating keratoplasty was done in 2 patients and 1 patient was lost in follow up. Out of 8 patients who developed corneal perforation, in 3 patients temporary tarsorrhaphy was done, 2 patients therapeutic penetrating keratoplasty was done. Evisceration was done in 1 patient and 2 patients were referred outside for therapeutic penetrating keratoplasty. Anatomical integrity was achieved in all four eyes which under went therapeutic penetrating keratoplasty but their visual outcome was poor. In three patients, reinfection of the corneal graft occurred which healed after treatment with leucomatous opacity and vasculasization. In one patient grafted there was no reinfection but vision was only finger counting close to face.

\section{VISUAL OUTCOME}

Vision was improved with treatment in $30(50 \%)$ of patients. Majority of the patients $(35,58.33 \%)$ were having vision between finger counting to no perception of light. We looked for any association of treatment outcome (in form good outcome and poor outcome) with sex of patients, residence of patients, presence or absence of ocular trauma, size of corneal ulcer, depth of corneal ulcer, grade of corneal infiltrates, presence or absence of hypopyon, Gram stain, Giemsa staining, $\mathrm{KOH}$ mount, growth in culture. By good outcome we meant, complete healing of corneal ulcer and with poor outcome we meant nonhealing or perforation of corneal ulcer.

Similarly we looked for any association in occurrence of corneal perforation with sex of patients, residence of patients, presence or absence of trauma, size of corneal ulcer, depth of corneal ulcer, grade of corneal infiltrates, presence or absence of hypopyon, Gram stain, Giemsa staining, $\mathrm{KOH}$ mount, growth in culture. In our study factors like gender of patients( $p$ $0.588)$, patient's residence( $\mathrm{p} 0.614)$, ocular trauma( $\mathrm{p} 0.517)$, previous treatment( $\mathrm{p} 0.349)$, presence or absence of hypopyon(p 0.215$)$ were not associated with poor treatment outcome or corneal perforation. There was statistically significant association of factors like size of corneal ulcer( $p$ $0.003)$, depth of corneal ulcer(p 0.000), grade of corneal infiltrates(p 0.000), Gram stain(p 0.008), KOH stain(p 0.017), and microbial growth during culture(p 0.028), with poor treatment outcome.

\section{DISCUSSION}

Suppurative keratitis was mostly seen in middle age male patients. The mean age of the patients was 41.53 years ranging from 5-83 years. In the study done by M R Shoja et al, the mean 
age was $45.3 \pm 18.5$ years (range 1-79 years), the highest frequency belongs to the 40 to 50 age group, and $70 \%$ of the cases were under 60 years of age. ${ }^{12}$ Presentation of corneal ulcer in this age being more common due to the fact that persons belonging to this age group are more active and involve themselves in outdoor activities. An other factor could be that they are also the earning member of the family so are brought more frequently to the hospital. ${ }^{13}$ In our study male were affected approximately two times more (65\%) commonly than female (35\%). Similar result was found in a study done by M J Bharathi et al, male $(65.02 \%)$ were more often affected then female (34.98\%) and large proportion of the patients $(66.85 \%)$ were in the younger age group (21 to 50 years). ${ }^{14}$ Our hospital is situated at the foothill of the mighty Himalayas in the Eastern Region of Nepal. It is a tertiary care centre which provides referral services to the 16 districts of eastern Nepal and also neighboring states of India like Bihar and West Bengal. Most of the patients were from planes of Nepal and India whereas only 5 patients $(8.33 \%)$ were from hilly areas. This can be explained by the pleasant cold climate and poor road network in the hills. Majority of population live in rural areas of Nepal, so majority of the patients were from rural areas. Forty four (73.3\%) were from rural areas and 16 (26.7\%) were from urban areas. In a study done by M R Shoja et al in Ireland, sixty-five percent were of urban and 35\% from rural areas. ${ }^{12}$

\section{PREDISPOSING FACTORS}

In the developing countries like Nepal and India, agriculture is the single most common occupation of the adult population involving both the sexes. Agricultural workers (33.67\%), housewife $(28.33 \%)$ and students (15\%) were the three most common group comprising $48(80 \%)$ of our total study population. There was history of ocular trauma in $35(58.33 \%)$ patients in our study and in $25(41.67 \%)$ patients no predisposing factor was found. Vegetative trauma during agricultural work was the commonest mode of trauma accounting 15 (42.86\%) of ocular trauma. In a study done by M Srinivasan et al in India in 1997 on 434 patients of corneal ulcers, the majority of the patients were farmers or hired agricultural workers, usually working in rice and sugar cane fields $(56.4 \%)$ followed by house wife $(12 \%){ }^{15}$

Mean duration of illness was 20.23 days ranging from 2 to 90 days. It was long as patients presented late mostly after taking some treatment from their local practiceners. In the study done in 2007 by A Panda et al, majority of the patient presented between 2 to 5 weeks after the onset of the symptoms. ${ }^{16}$ Similarly Basak and colleagues (2005) identified ocular trauma with vegetative or organic materials to be the most common predisposing factor. A history of recent corneal injury was obtained in $994(82.9 \%)$ patients. $715(59.6 \%)$ patients had corneal injury with vegetative matter; mostly (526; $43.9 \%$ ) paddy or paddy stalk $(\mathrm{P}<0.0001)$, followed by jute plant $(128 ; 10.6 \%)$. Other significant agents were twig of a tree, flying insect, dirt, mud, sand, etc. ${ }^{9}$ In developed countries use of soft contact lenses has greatly increased the risk of suppurative keratitis In the study done by T Bourcier et al in France in 2003, contact lens wear was the most common risk factor which was encountered in 50.3\%. Soft contact lens was noted in $89.4 \%$ of cases, rigid gas permeable contact lens in $8.6 \%$ and hard PMMA contact lens in $2 \%$ of cases. ${ }^{17}$ In the study carried by F Schaefer et al (2001) in Switzerland on 85 cases of bacterial keratitis, contact lens wear was associated in $31(36 \%)$ cases. Ocular trauma was the second most common cause of bacterial keratitis accounting $17(20 \%)$ of cases. ${ }^{18}$

\section{CLINICAL FEATURES}

Majority of our patients 49 (81.67\%) have already received treatment from outside. In the tertiary care centers, most of the patients are referred by the general practitioners or ophthalmologist after primary treatment. In the study done by T. Bourcier el al (2003) 72 (24\%) patients were already being treated with topical antibiotics. ${ }^{17}$ In the study done in 2007 by A panda et al, the majority of the patients (970/1000) either were referred by general practitioners or ophthalmologists, or were patients who presented themselves as they were not noticing any improvement with the treatments. ${ }^{16}$

Most common position of ulcers was central which was found in $37(61.7 \%)$ of the patients; followed by inferior, seen in 12 $(20 \%)$ of the patients. Temporal position was found in 7 $(11.7 \%)$ patients, superior and nasal position was seen in 3 $(5 \%)$ and $1(1.6 \%)$ patients. in the study done by A Saeed et al location of corneal infiltrates was central 69.0 (76.7\%), peripheral $21.0(23.3 \%)$ and hypopyon was present in 23.0 $(25.6 \%)^{13}$

\section{MICROBIOLOGICALPROFILE}

The epidemiological pattern and causative agents for suppurative corneal ulcer varies significantly from country to country, and even from region to region within the same country. Staphylococcus sp was assumed to predominate in 
cool and desert climate and Pseudomonas aeruginosa and fungi isolate were thought to be more common in the warm and dry climates. Staphylococcus species is found to be more common isolate in most of the series on bacterial keratitis.

In our study out of 60 patients of suppurative corneal ulcer analyzed during culture growth was seen in $33(55 \%)$ patients. Fifteen cases (25\%) had a pure bacterial growth, $10(16.33 \%)$ patient had pure fungal growth and $8(13.33 \%)$ cases had mixed bacterial and fungal growth. Staphylococcus aureus was isolated in $15(25 \%)$ cases, among them in $4(6.67 \%)$ cases it was single isolate and in 11(18.33\%) it was a mixed growth along with fungus. Among fungi Aspergillus flavus, Aspergillus fumigatus and Fusarium were the frequent isolates, found in $4(6.67 \%), 3(5 \%)$ and $3(5 \%)$ cases respectively.

In the study done by F. Schaefer et al The most commonly isolated bacteria were Staphylococcus epidermidis, 40\%; Staphylococcus aureus, 22\%; Streptococcus pneumoniae, $8 \%$; others Streptococcus species, 5\%; Pseudomonas, 9\%; Moraxella and Serratia marcescens, 5\% each; Bacillus, Corynebacterium, Alcaligenes xyloxidans, Morganella morganii, and Haemophilus influenza, $1 \%$ each. ${ }^{18}$ In the study done by Basak et al culture were positive in $811(67.7 \%)$ of total corneal ulcer in which $509(42.5 \%)$ patients have pure fungal growth, $184(15.2 \%)$ had pure bacterial growth and $114(9.5 \%)$ had mixed growth. Staphylococcus aureus was the most commonly isolated bacteria $127(42.6 \%)$ of all positive bacterial culture. Other isolated gram positive bacteria were Staphylococcus epidermidis (15.7\%), Streptococcus pneumoniae (9.4\%) and Diptheroids. Gram negative bacteria were Pseudomonas 63 (21.1\%) and Enterobacter species, Moraxella and Hemophilus influenzae. There were 623 fungal isolates in 509 patients - 373 (59.8\%) grew Aspergillus species, 132 (21.2\%) grew Fusarium species and 63 (10.1\%) grew Penicillium species. Yeast form, Candida species was positive in $7(1.1 \%)$ cases. Dematicious fungi, such as Curvularia, Alternaria, Bipolaris and Cladosporium were present in $12(1.7 \%)$ cases. ${ }^{9}$ In the study done by MP Upadhyay (1991) microorganisms were grown from 324 (80\%) of the ulcers. Pure bacterial cultures were obtained from $256(63.2 \%)$ of the patients, whereas pure fungal cultures were obtained from 27 (6.7\%) of the patients. In 41 patients (10.1\%), corneal cultures yielded a mixed growth of bacteria and fungi. Of a total of 398 bacterial isolates, 124 (31.1\%) were positive for Streptococcus pneumoniae, the most commonly isolated organism in the series. Other frequently isolated bacteria included Staphylococcus epidermidis, S. aureus, and Pseudomonas species. Of 68 positive fungal isolates obtained, $32(47.0 \%)$ were identified as Aspergillus species. Candida and Fusarium species were less commonly seen. ${ }^{4}$

Fungi are commonly isolated in the culture. This can be explained by the fact that most patients had already received antibiotic which may have killed the bacteria of the corneal ulcers but could not affect the fungi. Aureobasidium pullulans is considered to be a rare entity and so far very few reports have appeared in the world literature but it is isolated regularly from our centre. In our study Auerobasidium was isolated in 2 (3.33\%) patients. A Panda et al analyzed 447 patients with presumed microbial keratitis from 1 August 1998 to 31 July 2001 retrospectively. Aureobasidium pullulans was found in 25 cases. $^{16}$ Similarly in the study done by B Khanal et al involving 86 patients from june 1998 to july 1999, Aureobasidium pullulans was found in 4 cases. ${ }^{19}$

\section{TREATMENT OUTCOME}

In our study of 60 patients, $49(81.67 \%)$ patients achieved good out come in form of complete healing of corneal ulcer. Poor outcome was seen in $11(18.33 \%)$ patients. Among them ulcer was nonhealing in $3(5 \%)$ patients and corneal perforation occurred in $8(13.33 \%)$ patients. In the study done by BR Keshav et al 130 (69.14\%) patients recovered, 18 $(9.57 \%)$ patients showed signs of regression but failed to keep up the appointment to assess the course of the ulcer, 10 (5.31\%) patients the ulcer remained stationary and left seeking advice elsewhere, 17 (9\%) patients left against medical advise and 9 cases $(4.78 \%)$ worsened. Perforation at the time of presentation or after starting treatment in the hospital was seen in $33(17.55 \%)$ patients. $^{20}$

In our study factors like gender of patients, patient's residence, ocular trauma, previous treatment, presence or absence of hypopyon were not associated with treatment outcome or corneal perforation. There was statistically significant association of factors like size of corneal ulcer, depth of corneal ulcer, grade of corneal infiltrates, Gram stain, $\mathrm{KOH}$ stain and microbial growth during culture with poor treatment outcome. In the study done by $\mathrm{N}$ morlet et al significant predictors of slow healing were previous ocular disease and a positive culture and significant predictors of indolent ulceration were previous ocular disease and steroid use at diagnosis. A positive culture result alone was predictive of poor healing regardless of the organism obtained. ${ }^{21}$ 


\section{CONCLUSION}

Corneal ulcers are more commonly seen in middle aged male, involved in agriculture related work in rural areas. History of ocular trauma is seen in majority of the patients. Vegetative trauma is the most common predisposing factor. Microbial growthpositivity is seen in 55\% of patients with corneal ulcer. Staphylococcus aureus is the most common bacterial isolates and Aspergillus is the most commonly isolated fungal species. Good out come in form of complete healing of corneal ulcer with treatment was seen in $81.67 \%$ of patients. Factors like size of corneal ulcer, depth of corneal ulcer, grade of corneal infiltrates and microbial growth during culture are associated with poor treatment outcome.

\section{REFERENCES}

1. Resnikoff S, Pascolini D, Elya'ale D. Global data on visual impairment in the year 2002. Bull World Health Org 2004;82:84455.

2. Whitcher JP, Shrinivasan M. Corneal ulceration in the developing worlda silent epidemic. $\mathrm{Br} J$ Ophthalmol 1997;81:6223.

3. Whitcher JP, Shrinivasan M, Upadhyay MP. Corneal blindness: a global perspective. Bull World Health Organ 2001;79:21421.

4. Upadhyay MP, Karmacharya PC, Koirala S, Tuladhar NR, Bryan LE, Smolin G. Epidemiologic characteristics, predisposing factors, and aetiologic diagnosis of corneal ulceration in Nepal. Am J Ophthalmol 1991;111:92-99.

5. Erie JC, Nevitt MP, Hodge DO. Incidence of ulcerative keratitis in a defined population from 19501988. Arch Ophthalmol 1993;111:166571.

6. Jeng BH, McLeod SD. Microbial keratitis. Br J Ophthalmol 2003;87:805-806.

7. Upadhyay MP, Karmacharya PC, Koirala S. The Bhaktapur Eye Study: ocular trauma and antibiotic prophylaxis for the prevention of corneal ulceration in Nepal. Br J Ophthalmol 2001;85:38892.

8. Ormerod LD, Hertzmark E, Gomez DS. Epidemiology of microbial keratitis in Southern California. A multivariate analysis. Ophthalmology 1987;94:1322-33.

9. Basak SK, Basak S, Mohanta A, BhowmickA. Epidemiological and Microbiological Diagnosis of Suppurative Keratitis in
Gangetic West Bengal, Eastern India. Indian J Ophthalmol 2005;53:17-22

10. Kaufman HE, Barron BA, McDonald MB. The Cornea Churchill Livingstone, New York, USA, 1988;217-247.

11. Performance standards for antimicrobial disk susceptibility tests, $9^{\text {th }}$ ed. Approved standard M2-A9. Clinical and Laboratory standards institute. 2006, Wayne, Pa.

12. Shoja MR, Manaviat M. Epidemiology and outcome of corneal ulcer in Yazd Shahid Sadoughi Hospital. Acta Medica Iranica. 2004;42(2):136-141.

13. Saeed A, D'Arcy F, Stack J, Collum LM, Power W, Beatty S. Risk factors, microbiological findings, and clinical outcomes in cases of microbial keratitis admitted to a tertiary referral center in ireland. Cornea. 2009; 28(3):285-92.

14. Bharathi MJ, Ramakrishnan R, Vasu Samala, Meenakshi R, Palaniappan R. Epidemological characteristics and laboratory diagnosis of fungal keratitis. A three-year study. Indian J Ophthalmol 2003;51:315-21.

15. Shrinivasan M, Gonzales CA, George C, Cevallos V, Mascarenhas JM, Asokan B, Wilkins J, Smolin G, Whitcher JP. Epidemiology and aetiological diagnosis of corneal ulceration in Madurai, South India. Br J Ophthalmol 1997;81:965-971.

16. Panda A, Satpathy G, Nayak N, Kumar S, Kumar A. Demographic pattern, predisposing factors and management of ulcerative keratitis: evaluation of one thousand unilateral cases at a tertiary care centre. Clinical and Experimental Ophthalmology 2007;35:4450.

17. T Bourcier, F Thomas, VBorderie, C Chaumeil, and L Laroche. Bacterial keratitis: predisposing factors, clinical and microbiological review of 300 cases. Br J Ophthalmol. 2003; 87(7):834-838.

18. Schaefer F, Bruttin O, Zografos L, et al. Bacterial keratitis: a prospective clinical and microbiological study. $\mathrm{Br} J$ Ophthalmol 2001;85:8427.

19. Khanal B, Kaini KR, Deb M, Badhu B, Thakur SK. Microbial keratitis in eastern Nepal. Trop Doct. 2001 Jul;31(3):168-9.

20. Keshav BR, Zacheria G, Ideculla T, Bhat V, Joseph M. Epidemiological Characteristics of Corneal ulcers in south sharqiya Region. Oman Medical Journal 2008;23(1).

21. Morlet N, Minassian D, Butcher J. Risk factors for treatment outcome of suspected microbial keratitis. Ofloxacin Study Group. Br JOphthalmol. 1999;83(9):1027-31. 